\title{
The best marker for guiding the clinical management of patients with raised intracranial pressure - the RAP index or the mean pulse amplitude?
}

\author{
Allan Hall ${ }^{1} \cdot$ Roddy O'Kane $^{2}$ \\ Received: 9 May 2016 / Accepted: 8 August 2016/Published online: 27 August 2016 \\ (C) The Author(s) 2016. This article is published with open access at Springerlink.com
}

\begin{abstract}
Raised intracranial pressure is a common problem in a variety of neurosurgical conditions including traumatic brain injury, hydrocephalus and intracranial haemorrhage. The clinical management of these patients is guided by a variety of haemodynamic, biochemical and clinical factors. However to date there is no single parameter that is used to guide clinical management of patients with raised intracranial pressure (ICP). However, the role of ICP indices, specifically the mean pulse amplitude (AMP) and RAP index [correlation coefficient (R) between AMP amplitude (A) and mean ICP pressure (P); index of compensatory reserve], as an indicator of true ICP has been investigated. Whilst the RAP index has been used both as a descriptor of neurological deterioration in TBI patients and as a way of characterising the compensatory reserve in hydrocephalus, more recent studies have highlighted the limitation of the RAP index due to the influence that baseline effect errors have on the mean ICP, which is used in the calculation of the RAP index. These studies have suggested that the ICP mean pulse amplitude may be a more accurate marker of true intracranial pressure due to the fact that it is uninfluenced by the mean ICP and, therefore, the AMP may be a more reliable marker than the RAP index for guiding the clinical management of patients with raised ICP. Although further investigation needs to be undertaken in order to fully assess the role of ICP indices in guiding the clinical management of patients with raised ICP, the studies
\end{abstract}

Allan Hall

Allan.Hall.2@glasgow.ac.uk

1 School of Medicine, University of Glasgow, University Avenue, G12 8QQ Glasgow, UK

2 Department of Neurosurgery, Institute of Neurological Sciences, Queen Elizabeth University Hospital, G51 4TF Glasgow, UK undertaken to date provide an insight into the potential role of ICP indices to treat raised ICP proactively rather than reactively and therefore help prevent or minimise secondary brain injury.

Keywords Intracranial pressure $\cdot$ RAP index $\cdot$ Mean pulse amplitude

\section{Introduction}

The accurate measurement of dynamic intracranial pressure is complex. Although the measurement of ICP is an important factor in determining both the clinical management and outcome of patients with several neurosurgical conditions, recent studies have looked at the use of measuring the compensatory reserve as a possible predictor of clinical outcome and how it could be used to guide clinical management. Czosnyka et al. designed a coefficient between the mean intracranial pressure and the ICP mean pulse amplitude, the RAP index, as a potential descriptor of neurological deterioration in traumatic brain injury (TBI) patients [1]. Since this publication it has also been used to characterise the compensatory reserve in patients with hydrocephalus [2]. More recent studies have researched the use of the RAP index as a possible marker to guide clinical management in patients with raised ICP. This has included analysing the effects of baseline effect errors on both the RAP index as well as the ICP wave parameters, including the ICP mean pulse amplitude (AMP) [3, 4]. These studies concluded that baseline effect errors may hinder the use of the RAP index as a marker to guide clinical management due to their influential effect on the mean ICP, which is one of two factors used to calculate the RAP index. Other studies have shown that the AMP, as opposed to the RAP index, is refractory to these baseline errors and may provide a more accurate marker to guide clinical management, 
as well as its potential to predict mortality in certain neurosurgical events including traumatic brain injury [5].

This review will give an overview of intracranial pressure including its monitoring and examine the evidence from previous studies regarding the potential role of both the RAP index and the AMP as a marker to guide clinical management.

\section{Intracranial pressure}

\section{Physiology}

Intracranial pressure (ICP) is derived from the circulatory dynamics of the CSF and cerebral blood flow that occur within the rigid intracranial compartment [6]. This relationship forms the ICP waveform, which consists of a cardiac component, which involves three peaks, a respiratory component, which is a reflection of the respiratory cycle on cerebral venous blood flow, and slow vasogenic waves of ICP, secondary to autoregulation-derived cyclic fluctuations of arterial blood volume (Fig. 1). ICP is affected by many factors both within and outside of the intracranial compartment.

Under normal circumstances ICP is kept within a certain range primarily with cerebral auto-regulation, which is itself dependent on cerebrovascular reactivity. The fact that true ICP is influenced by many factors and has both inter- and intra-patient variability makes accurate measurement of true ICP difficult. For example, the value of ICP is affected by age, body posture, time of day as well as the clinical condition of the patient. However a normal range of ICP has been established in order to address the multi-variant nature of ICP, as well as general interventional thresholds for ICP in different neurosurgical conditions.

Intracranial factors that can affect ICP can be broadly categorised into three main intracranial areas: disturbances in the dynamics of CSF; the expansion of intracranial tissue itself; the expansion of blood volume (Fig. 2). Disturbances within these intracranial areas can be caused by several factors including disturbances in the normal vascular architecture (resulting in either haemorrhage or mass effect), infection, malignancy (including metastasis) and trauma.

Extra-cranial factors are diverse and more difficult to manage from a neurosurgical point of view. For example, both the intra-thoracic compartment and, to a lesser extent, the intra-abdominal compartment influence ICP, in both the acute and chronic setting. In particular, the respiratory system plays a crucial role in the cerebral circulatory dynamics. This is evident by the role of ventilation to help reduce the vasodilatory effects of $\mathrm{CO}_{2}$ in patients with TBI $[7,8]$.

\section{Measurement and monitoring}

Although understanding the homeostasis of ICP and its relationship to intracranial events is vital, the accurate measurement of true ICP is crucial in understanding its role in abnormal intracranial physiology and its effect on clinical outcome.

Measuring ICP is conventionally undertaken using either an intra-parenchymal or intra-ventricular transducer. It has been hypothesised that measuring intracranial pressure from a single source is itself a source of inaccuracy due to the complex three-dimensional pressure gradients that are established as a result of the complex intracranial architecture and physiology (Fig. 3). Studies have shown there is regional cerebral homeostasis as a result of regional effects on vasculature from local factors including metabolic and neural factors [9]. This local effect on regional vasculature is driven by cellular events ranging from increased production of waste products from cellular metabolism to changes in local neuronal activity. The reactivity of cerebral blood flow (CBF) to metabolic demand is not only rapid but is not always proportional [9]. This regional homeostatic effect has the potential to be another factor that could affect the true ICP values when measuring the ICP from a single source. However due to the invasive nature of ICP monitoring, accurately measuring pancranial ICP using the current invasive methods would be impractical.

Non-invasive methods of monitoring ICP have been assessed, including MRI and ultrasonography [10]. In particular the use of ultrasonography to measure optic nerve sheath diameter to assess dynamic changes in ICP has shown promising results [11]. Although no method has currently been found to be a suitable substitute for the more invasive method of ICP monitoring [12], further study into its use as a method of monitoring ICP from a variety of intracranial points would be useful, in particular, the potential role of using imaging techniques including fMRI to assess cerebral function and thereby establish the full relationship between raised ICP and changes in cerebral function. However the heterogeneity of cerebral vascular reactivity caused by ischaemia-induced impairment of cerebrovascular dynamics, including neurovascular coupling, could potentially hinder the use of fMRI in accurately assessing cerebral function in conditions that disrupt the blood-brain barrier such as in TBI.

\section{Intracranial pressure indices}

\section{The RAP index}

Czosnyka et al. designed an index that is a correlation coefficient between the mean ICP and the ICP mean wave amplitude (AMP) - the RAP index [correlation coefficient (R) between AMP amplitude (A) and mean pressure (P); index of 
a (i)

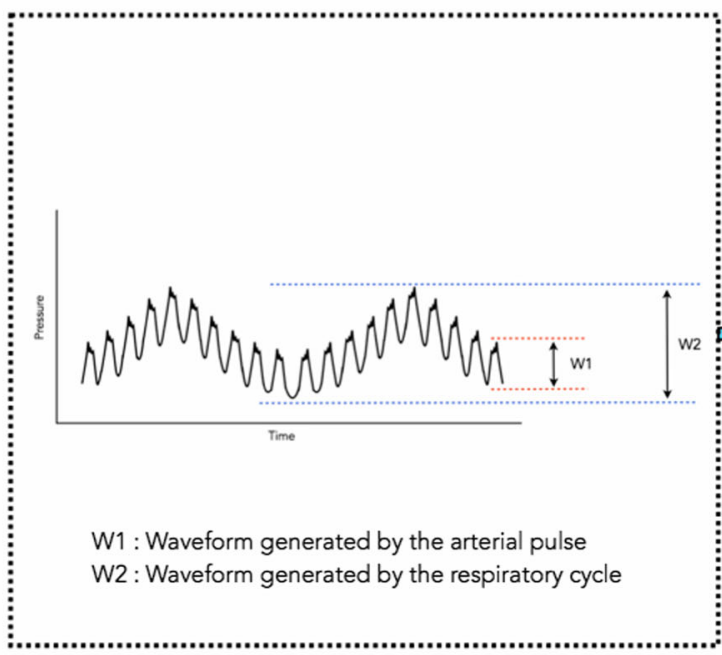

b
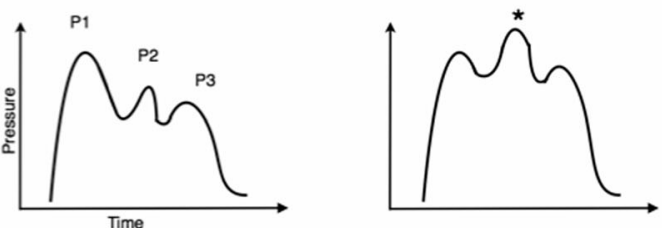

Normal intracranial physiological conditions a (ii)

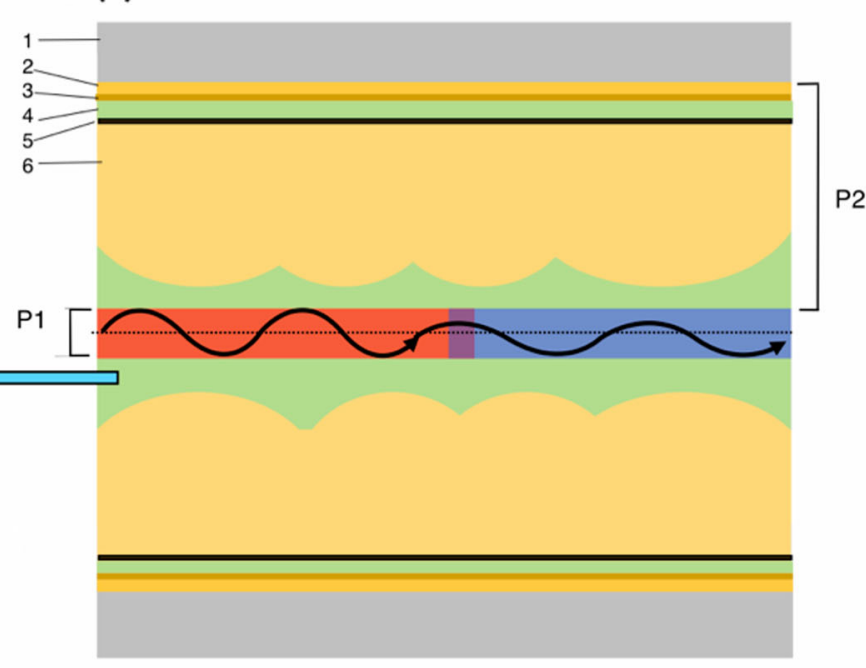

C

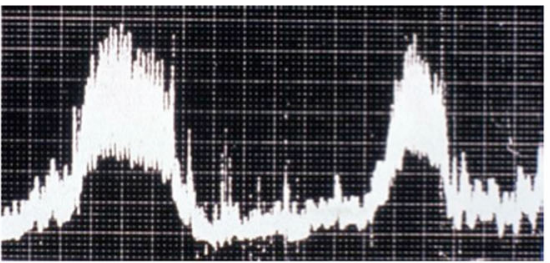

A waves (plateau waves)

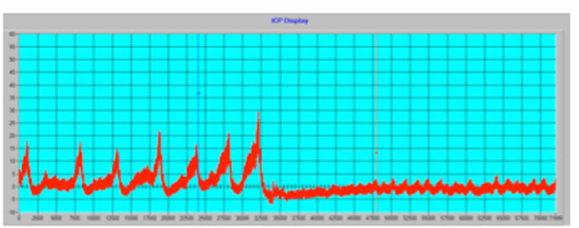

B waves
Fig. 1 An illustration of the ICP waveform components. a (i) The ICP waveform showing the cardiac component (W1) and respiratory component (W2). The cardiac component consists of three peaks: P1 (reflects arterial pulsation), P2 (reflects, to a degree, intracranial compliance) and P3 (reflects aortic valve closure). The respiratory component reflects the cerebral venous pulsation generated by the changes in intra-thoracic pressure that is generated by respiration. a (ii) An illustration of what the individual ICP wave form peaks represent in terms of intracranial physiology. The black oscillating arrow represents arterial pulsations (red area), which subsequently become venous pulsations (blue area) as cerebral blood travels through the cerebral vasculature. $\mathrm{P} 1$ is the pressure generated in the CSF as a result of the pressure generated from the arterial pulsation. $\mathrm{P} 2$ is the pressure generated within the CSF as a result of the forces generated from both the pulsatile blood vessel and the resistance from the intracranial tissue. The higher the resistance is, the greater the pressure within the CSF and therefore the greater the $\mathrm{P} 2$ wave amplitude. Note the intracranial compliance, and therefore the value of $\mathrm{P} 2$, can be affected by any factor from the endothelium of the cerebral blood vessel to the intracranial surface of the skull (represented by P2) Diagram key: 1, skull; 2, dura layer; 3, arachnoid layer; 4, subarachnoid space containing the cerebrospinal fluid (represented in green); 5, pia layer; 6, cerebral tissue. b The individual ICP waveform peaks. Under the normal intracranial physiological state, $\mathrm{P} 1>\mathrm{P} 2>\mathrm{P} 3$. However when ICP is raised, and there is reduced intracranial compliance, $\mathrm{P} 1<\mathrm{P} 2>\mathrm{P} 3$. $\mathrm{c}$ The slow vasogenic waves of ICP, secondary to autoregulation-derived cyclic fluctuations of arterial blood volume. A waves, or plateau waves, always indicate pathology and signify reduced intracranial compliance. Once they are present, the patient will likely have neurological deterioration. They involve a steep rise in ICP from around a normal value to $50 \mathrm{mmHg}$ or more. They persist for 5-20 min after which there is a sharp decline. B waves are more subtle than A waves and can indicate several abnormalities of intracranial homeostasis including being an indicator of failing intracranial compensation. It is important to note however that they can also be present in patients with normal ICP. B waves are rhythmic oscillations with ICP rising in a crescendo manner to levels 20-30 mmHg higher than baseline, with a subsequent sharp decline. They occur every 1-2 min. Note, Lundberg A waves and B waves refer to raises in static (mean ICP) and are not related to the pressure fluctuations during the cardiac cycle, which is the case for ICP wave amplitudes 


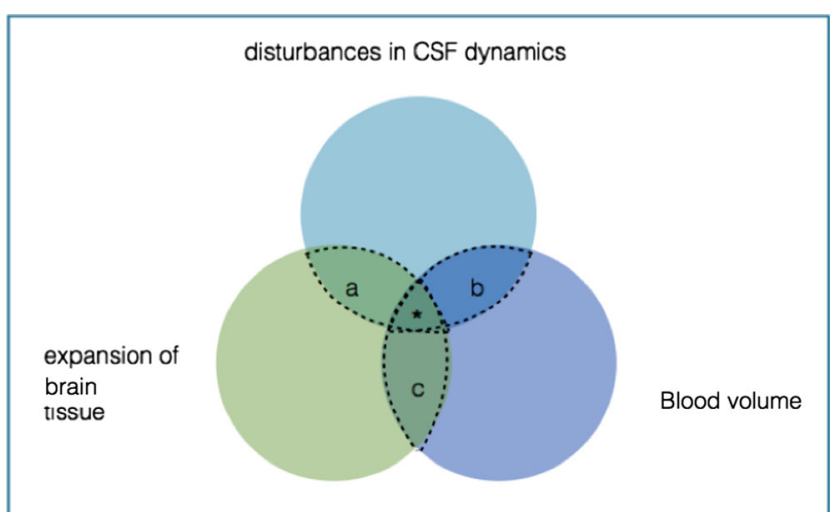

Fig. 2 Intracranial factors that affect ICP: the Monro-Kellie Doctrine. Each circle represents one of the three broad intracranial categories: blood, CSF and brain tissue. If the normal physiological and/or anatomical state of either one of these categories is altered, and the compensatory change from the other categories is exhausted, this will result in an increase in ICP. The overlap of the circles represents how each category can affect the other $(\mathrm{a}, \mathrm{b}, \mathrm{c})$. The density of the circles correlates with the ICP, with * denoting the highest ICP

compensatory reserve]. This was first introduced as a potential descriptor for neurological deterioration in TBI patients. It was subsequently used to characterise the pressure-volume compensatory reserve in patients with hydrocephalus. The RAP index has been shown to be a reliable measure of the compensatory reserve; however in more chronic states the cerebrospinal elasticity coefficient (E1) and AMP have been shown to express the compensatory reserve better [13-16]. This is due to the fact that raised ICP in chronic states, such as hydrocephalus and intracranial hypertension, is due to increased cerebral venous outflow or increased resistance to CSF outflow rather than the exhaustion of the cerebrospinal compensatory reserve. The RAP index has also been shown to be an accurate measure of intracranial compliance [17]. In addition, the RAP index has been used to assess the use of extra-ventricular devices to investigate the role of cerebral auto-regulation disturbances in aneurysmal subarachnoid patients [18].

The numerical value of the RAP index can range from +1 to -1 (Fig. 4). An RAP value of 0 is interpreted as lack of synchronisation between the changes in AMP and mean ICP. In terms of the pressure-volume relationship, an increase in intracranial volume does not result in a rise in ICP.

When the RAP index rises to +1 , there is synchronisation between the rise in AMP and the rise in ICP. In terms of the pressure-volume relationship, a small rise in intracranial volume results in a larger rise in ICP.

If the ICP continues to rise, the RAP index value becomes 1. At this point the AMP has an inverse relationship to ICPthe AMP decreases as the ICP continues to rise. At this stage the compensatory reserve is exhausted and cerebral autoregulation collapses. It is at this point that cerebral ischaemia- induced irreversible brain damage will likely occur and, ultimately, brain herniation. Note however that cerebral ischaemia will rise in a gradient fashion as the ICP continues to rise.

More recent studies have researched the potential role of the RAP index as a marker to guide clinical management [2, $19,20]$. However these studies found that the use of RAP as a potential marker to guide clinical management may be

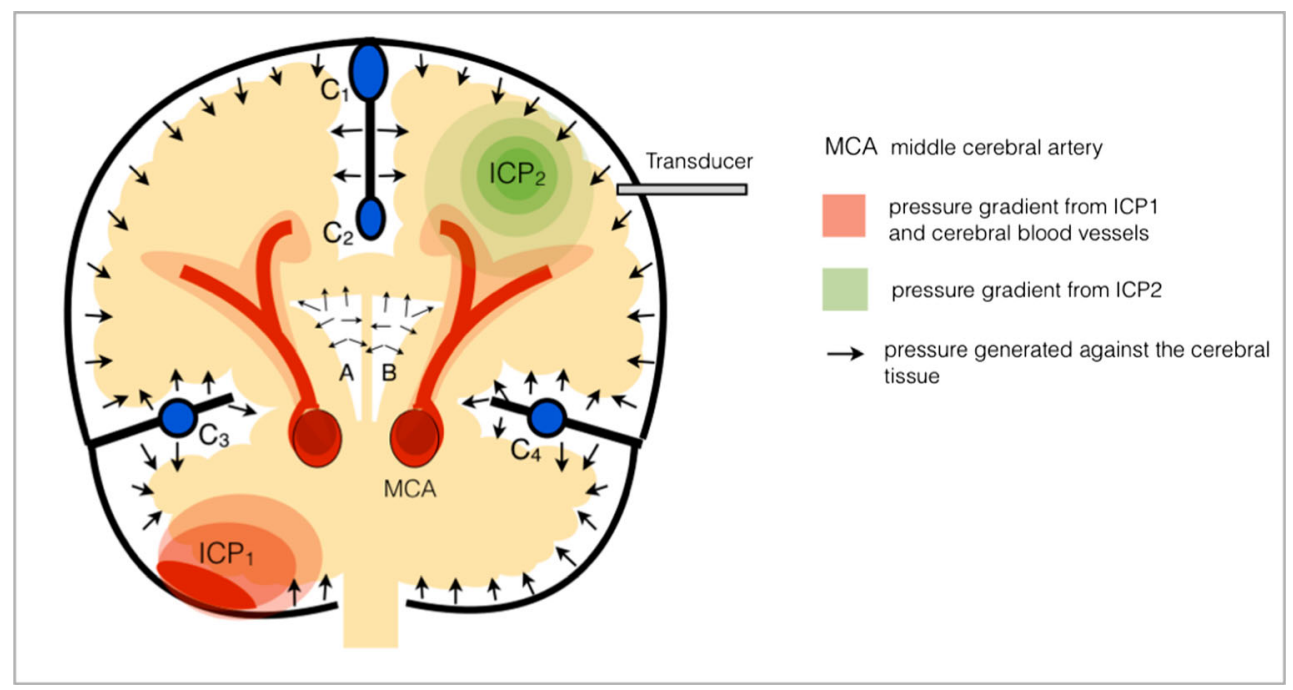

Fig. 3 The effect of pressure gradients on ICP monitoring. If the transducer is placed in the frontal-parietal region, as shown, the effect of the pressure gradients generated by event 1 (ICP1), for example cerebellar haematoma within the infra-tentorium, has the potential to be different from the effect of the pressure gradient that is generated by event 2 (ICP2), for example TBI. The three-dimensional pressure gradients generated against the cerebral tissue itself is what will ultimately affect cellular function - the core issue in raised ICP. Note that the cerebral blood vessels also generate pressure gradients, which are a result of both cerebral blood flow and cerebral blood volume, and are influenced by cerebral vascular reactivity. Diagram key: $C 1$, superior sagittal sinus; $C 2$, inferior sagittal sinus; $C 3$ and $C 4$, transverse sinus, $A$ and $B$, lateral ventricles 
Fig. 4 The relationship among the pressure-volume curve, RAP index and cerebral auto-regulation. When the RAP index is close to 0 , cerebral auto-regulation remains intact and there is good compensatory reserve. As the ICP continues to rise and the RAP index approaches +1 , the compensatory reserve decreases; however cerebral auto-regulation remains. When the ICP reaches the critical value, cerebral auto-regulation is lost and there is no compensatory reserve

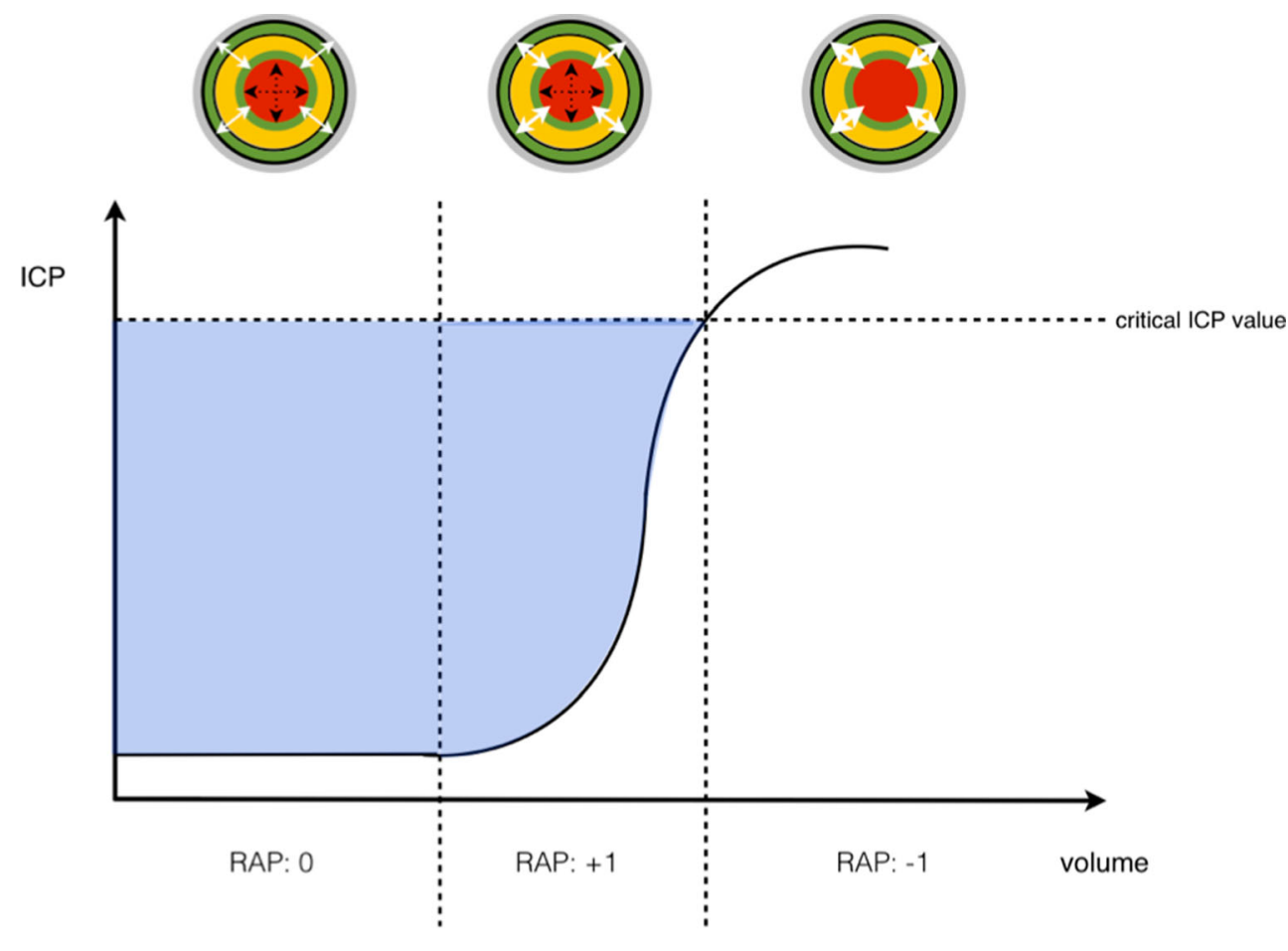

compensatory reserve

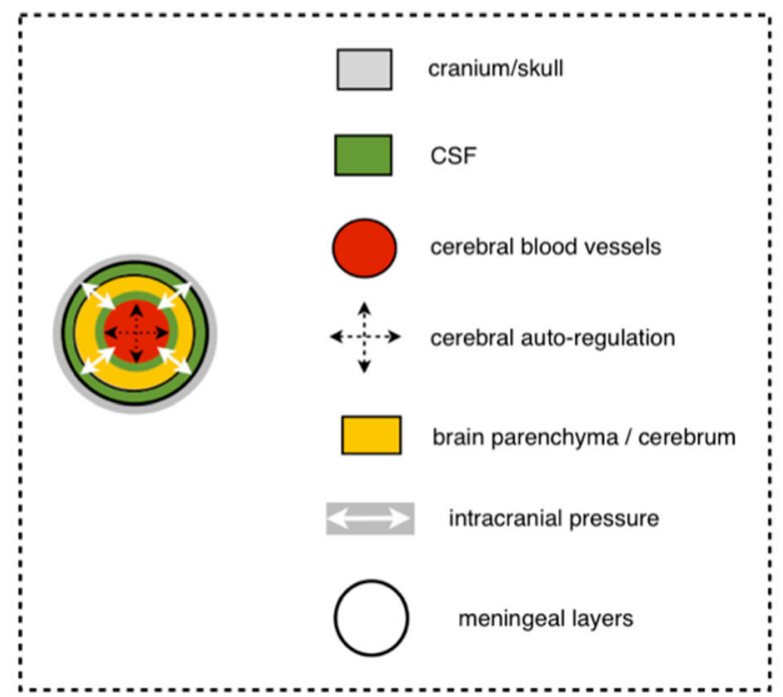

hindered by baseline effect errors. In particular it was found that baseline effect errors had a significant influence on the mean ICP as to render it an inaccurate reflection of the true ICP. This in turn influences the RAP index, which is calculated by correlating slow fluctuations of ICP and pulse amplitude.

\section{ICP mean pulse amplitude (AMP)}

The ICP mean pulse amplitude relates to the pressure fluctuations of the ICP during the cardiac cycle. The AMP increases as the ICP rises; however once the critical ICP has been reached there will be subsequent derangement of cerebrovascular reactivity and AMP will decrease.

More studies are beginning to research the use of AMP both as a more accurate marker of the true ICP, in place of the mean ICP, as well as a tool to help guide the clinical management of patients [21, 22]. The influence that baseline effect errors have been shown to have on the mean ICP has raised questions as to how accurately the mean ICP value reflects the true underlying ICP. In particular it has been shown that AMP can make a useful contribution to the 
diagnosis of hydrocephalus as well as predict a positive outcome after shunt insertion [23]. However its use as a single parameter to guide clinical management has not yet been justified.

Although it has been demonstrated that AMP is refractory to baseline effect errors, further study into what other factors could potentially affect AMP, and therefore its usefulness in clinical management, must be investigated. This includes investigating the extent to which any factor influences AMP across the different pathological conditions. For example, studies have shown that AMP is more dependent on pulse changes in arterial blood volume in patients with TBI than in those with normal pressure hydrocephalus [24].

Further studies have assessed the AMP in relation to clinical outcome and have shown that a lower AMP is associated with a better outcome following TBI [25] and surgery [26].

One proposed advantage of using AMP instead of the RAP index is its ability to reflect the dynamic nature of true ICP without influences from static values, which have the potential to be affected by other dynamic factors such as baseline effect errors. However one limitation of using the AMP is the ability of researchers to accurately analyse the multi-variant and dynamic nature of the ICP waveform in an objective way. Studies have looked at more sophisticated algorithms of objectively analysing ICP wave parameters, including AMP, with a degree of success.

For example, it has been shown that the time domain method is superior to the frequency domain method for ICP waveform analysis since the frequency domain method can underestimate the AMP in the presence of heart rate variability or if the waveform has a high harmonic distortion [27]. Holm et al. [27] showed that the difference in the AMP calculated from these two methods was significant, with a difference of $\geq 2.0 \mathrm{mmHg}$ in more than half of the 50,978 6-s time windows analysed. Furthermore, this difference will also affect the RAP index since the AMP is one of its components. Therefore, it is important that a standard method of analysis for the AMP is used in order to achieve robust results that can be accurately interpreted, which is important if both the RAP index and AMP are to be used to guide clinical management.

More recent studies have used more sophisticated algorithms to analyse the individual ICP waveform peaks ( $\mathrm{P} 1$, P2, P3) with a high degree of accuracy.

However the successful clinical use of such algorithms for accurate, objective analysis of Lundberg waves, in particular B waves, has still to be proven $[28,29]$. The proactive treatment of raised ICP instead of reactive treatment is one potential area where ICP waveform parameters, including AMP, could prove useful [30-32].

\section{Baseline effect errors}

Baseline effect errors (BEEs) are spontaneous shifts in baseline pressure that occur during continuous ICP monitoring. They were first described by Eide in 2006 [33] when monitoring ICP simultaneously from two separate sensors in which there was a marked difference in mean ICP, combined with close to identical ICP waveform components such as AMP.

Baseline effect errors can be caused by a variety of factors ranging from all components of the ICP monitoring system, including the sensor, cable, display and transducer, to imperfect fluid connection caused by debris or air bubbles.

In relation to ICP indices, this is very important since any ICP index that includes mean ICP in its component will be affected, including the RAP index. However since the AMP is included in the RAP index, the effect of BEE is less pronounced in the RAP index than in the mean ICP. Therefore, any ICP index that is solely based on static pressure measurements can expect to be affected by BEE.

The ability of baseline effect errors to influence the value of the RAP index, and therefore the true nature of the patients underlying intracranial compensatory reserve, has the potential to affect clinical management. It has been shown that significant differences in the RAP index correspond to the occurence of BEEs and that the differences are of a magnitude that could affect patient management [3]. For example, an inaccurate RAP index could give the clinician a false reflection of the patient's underlying intracranial physiological state and therefore instigate an erroneous change in their management that could range from altering doses of certain medications to undertaking inappropriate surgical intervention.

Baseline effect errors also have the potential to affect cerebral perfusion pressure, through the effect it has on intracranial pressure (since CPP is equal to mean arterial pressure minus intracranial pressure). For example, if BEEs are caused by an imperfect fluid connection as a result of debris or air bubbles, this could result in a fall in CPP if the ICP rises beyond the limits of cerebral auto-regulation.

\section{Current guidance in the clinical management of raised ICP}

Raised ICP is defined as a sustained ICP of greater than $20 \mathrm{mmHg}$. However the thresholds for management depend, in part, on the underlying cause. At present there is no single parameter that has been proven to be the best marker to guide the clinical management of raised ICP. Due to the multivariant nature of ICP between conditions and between patients it is doubtful whether it is possible to have one single parameter that can successfully guide the clinical management. The current treatment options for raised ICP range from initial medical management involving general physiological 
homeostasis to management, which involves more radical interventions including endotracheal intubation, barbiturate coma, and surgical evacuation or decompression. The ultimate goal of treating raised ICP is to prevent or minimise secondary brain injury by restoring intracranial homeostasis in order to reach optimum cerebral function.

\section{Treatment options}

\section{General physiological homeostasis}

General physiological homeostasis involves controlling several parameters, which include blood pressure, serum osmolarity, temperature and blood glucose, as well as maintaining adequate partial arterial pressures of oxygen.

Blood pressure control has an important role in the treatment of raised ICP; however the exact treatment thresholds required to achieve optimum cerebral function in the setting of raised ICP remains ambiguous. This is in part due to the fact that patients with raised ICP often have several contributing factors. An important consideration in the control of a patient's blood pressure within the context of raised ICP is the fine balance required between under-treatment of hypertension, and therefore increasing the possibility of haemorrhage or mass effect, and over-treatment of hypertension, and thus increasing the risk of ischaemia-induced damage to cerebral function. One therapy that aims to reduced the risk of ischaemia-induced cerebral damage is cerebral perfusion pressure (CPP)-guided therapy. This therapy aims to maintain CPP greater than $70 \mathrm{mmHg}$ in order to minimise ischaemia, which is induced by compensatory cerebral vasodilation [34-36]. However CPP-guided therapy is not conclusive as it has been shown that this approach can cause a rise in ICP [37].

Another approach used to control blood pressure is the "Lund Protocol" in which a disruption of the blood-brain barrier is assumed. This places emphasis on reducing the hydrostatic forces whilst increasing the osmotic forces to maintain fluid within the intravascular compartment and thus prevent vasogenic oedema [38].

In addition to ensuring adequate CPP, oxygen levels must be adequately maintained, particularly in patients who have suffered TBI or intracerebral haemorrhage (ICH) in which the presence of pericapillary oedema may reduce the diffusion ability of oxygen to cross the blood-brain barrier.

Another important therapeutic target in raised ICP is glycaemic control. It has been shown in several studies that hyperglycaemia increases both the morbidity and mortality in patients with several neurological conditions including ICH and traumatic brain injury (TBI) [39-42, 43, 44]. Several of the underlying reasons for this increase in morbidity and mortality are the widespread detrimental effect that hyperglycaemia has on the cardiovascular system. However it has also been shown that hyperglycaemia causes a rise in ICP [45]. In particular, the breakdown of the blood-brain barrier that occurs in TBI patients results in the influx of glucose into the cerebral tissue thereby causing an increase in oedema through osmosis.

Minimising pyrexic episodes in patients with raised ICP is very important as an increase in temperature results in a rise in the cerebral metabolic rate, which can precipitate ischaemia directly, or indirectly through oedema. This is evident by studies that have shown, through experimental models of brain injury, the detrimental effect fever has on clinical outcome $[46,47]$. Furthermore, the incidence of fever in patients who suffer basal ganglionic and lobar ICH, particularly ventricular haemorrhage, is high. Fever is also an independent predictor of poor clinical outcome after severe head injury. Therefore aggressive treatment of fever in patients with raised ICP is encouraged.

\section{Head of bed elevation}

Elevation of the head to $30^{\circ}$ improves jugular venous outflow and reduces ICP. However caution must be used in patients who are hypovolemic as elevation in these patients could result in a fall in CPP, which could have significant effects on cerebral function, depending on the volume and duration of the drop in CPP.

\section{Analgesia and sedation}

Analgesia is important in order to minimise the potential harmful effect on ICP. Sedation may be used in order to appropriately ventilate patients who are unable to achieve adequate ventilation on their own. However this must be titrated with care in order to minimise the detrimental effect it may have on the clinician's ability to clinically evaluate the patient.

\section{Neuromuscular blockage}

Patients who fail to respond to analgesia and sedation, and who still have intracranial hypertension, may benefit from neuromuscular blockade as seizure activity causes a rise in intra-thoracic pressure, which can obstruct cerebral venous outflow resulting in an increase in ICP. One of the drawbacks of this therapy is the masking of seizure activity.

\section{Osmotic agents}

One of the most important physiological properties that must be carefully balanced in patients with raised ICP is serum osmolarity. Both mannitol and hypertonic sodium chloride are the most common agents used.

Mannitol acts through two mechanisms. First, it increases intravascular volume and results in a reduction in blood viscosity. The intravascular volume expansion results in an increase in cardiac pre-load and thus an increase in CPP. 
Through cerebral auto-regulation, compensatory cerebral vasoconstriction occurs, which reduces the ICP. Second, mannitol draws water from the extracellular cerebral tissue into the plasma, thereby reducing cerebral oedema. However if the blood-brain barrier is disrupted, care must be taken to avoid worsening any cerebral oedema.

Hypertonic saline (HTS) can be used because of its osmotic effects as a result of its hypertonicity compared to extracellular cerebral tissue. However hypertonic saline also has haemodynamic, vaso-regulatory, immunological and neurochemical effects [48]. One recent study looked at the difference in cumulative and daily ICP burdens after severe traumatic brain injury between hypertonic saline and mannitol and found that HTS was more effective than mannitol when given as a bolus therapy. However the 2-week mortality rates between the two were not statistically different [49].

\section{Steroids}

The use of steroids in raised ICP is limited to treat vasogenic oedema where the underlying cause is primary and metastatic tumours. Steroids have shown no benefit in TBI or spontaneous intracerebral haemorrhage [50,51].

In some studies they have been shown to have a detrimental effect [52, 53].

Steroids can also be used in CNS infection; however care should be taken in intracerebral abscesses as they can retard the encapsulation process, worsen necrosis and reduce the permeability of antibiotics.

\section{Hyperventilation}

The use of $\mathrm{CO}_{2}$ influence on cerebral auto-regulation provides one of the most effective methods for rapidly lowering ICP. This is achieved through ventilation, which allows the clinician to lower $\mathrm{CO}_{2}$ levels and induce compensatory cerebral vasoconstriction and thus reduce the ICP. One caveat to using this property of $\mathrm{CO}_{2}$ is the ability of the extracellular space of the brain to accommodate the associated changes in $\mathrm{pH}$, and thus once $\mathrm{CO}_{2}$ levels begin to normalise a compensatory increase in ICP can occur. Therefore the main limitation of hyperventilation is its short therapeutic duration.

\section{CSF drainage}

CSF drainage is an effective method of lowering ICP in patients who have an intraventricular catheter to monitor ICP [31]. This is achieved by drainage of small amounts of CSF for short periods of time in response to elevations in the ICP. The main risks associated with ventriculostomy are infection and haemorrhage, with the incidence of bacterial meningitis varying between $6 \%$ and $22 \%[54,55,56]$.

\section{Barbiturate coma}

The use of barbiturates to induce a coma state is only suitable for refractory raised ICP. Barbiturates work by reducing the cerebral metabolic activity and thus reduce cerebral blood flow. However there are several potential side effects of this therapy including hypotension $[57,58]$. Therefore careful monitoring of the patient's haemodynamic state is vital.

\section{Surgery}

The role of decompressive craniotomy in the presence of a compressive pathology (such as subdural haematoma) is well documented. However its role in the absence of a compressive pathology is less well documented.

A number of studies, including the International Surgical Trial in Intracerebral Haemorrhage (STICH) trial, showed no real benefit in treating supra-tentorial ICH with craniotomy. Of those who were treated, a higher Glasgow Coma Scale $(\geq 9)$ and a clot within $1 \mathrm{~cm}$ of the surface were associated with a $29 \%$ relative benefit in functional outcome when compared with medical management [59-61]. Furthermore surgical intervention can provide more effective management in certain conditions such as diffuse, severe TBI and middle cerebral artery infarction than medical management [62].

However these studies did not include patients where the haemorrhage was located in the cerebellum. Further studies that did look at cerebellar ICH provided the conclusion that large cerebellar haemorrhages that either compress the brain stem or obstruct the fourth ventricle should be surgically removed as soon as possible [63, 64-69].

With regards to the timings of when surgery should be performed, there is no clear guidance on this [70]. The most supportive evidence is for operative removal within $12 \mathrm{~h}$; however this is not conclusive.

Furthermore, bifrontal decompressive craniectomy in posttraumatic intracranial hypertension has been associated with significant reductions in AMP and has increased the compensatory reserve and therefore led to an improvement in the patient's RAP index. [71] Although decompressive craniectomy has been reported to be beneficial in a number of conditions there is currently insufficient evidence to prove that it has the potential to improve outcome, despite showing benefits under certain conditions [72, 73].

\section{The role of the RAP index and AMP in future clinical management}

ICP indices were introduced to help improve the diagnostic value of ICP. One of the main reasons for using ICP indices in patients with raised ICP is to allow clinicians to treat raised ICP proactively rather than reactively. The RAP index has 
already been used as a descriptor of neurological deterioration in TBI patients. However it has yet to be used to guide clinical management. One of the main reasons the RAP index may not be a suitable marker to guide clinical management is its susceptibility to baseline effect errors. Given the dynamic nature of ICP in conjunction with the varying causes responsible for baseline pressure effects, it is little surprise that the use of a static value of ICP (the mean ICP) may not provide the most accurate measure of true, global ICP. One appeal of using AMP instead of the RAP index as a marker for guiding clinical management in patients with raised ICP is the fact that the AMP has been shown to be uninfluenced by baseline effect errors. However one limitation of using AMP, and other parameters of the ICP waveform, as a marker to guide clinical management is the lack of appropriate tools to accurately analyse the ICP waveform objectively. However more sophisticated algorithms are making this less of a problem, such as the MOCAIP (morphological clustering and analysis of continuous intracranial pressure) method [19], which has shown high accuracy in analysing several ICP waveform parameters [55].

It is clear from the literature to date that the use of either the RAP index or the AMP as a marker to guide clinical management has its limitations.

However given the complexity of ICP, and the heterogeneity of the intracranial conditions that it is affected by, it is difficult to imagine that the RAP index or AMP alone will

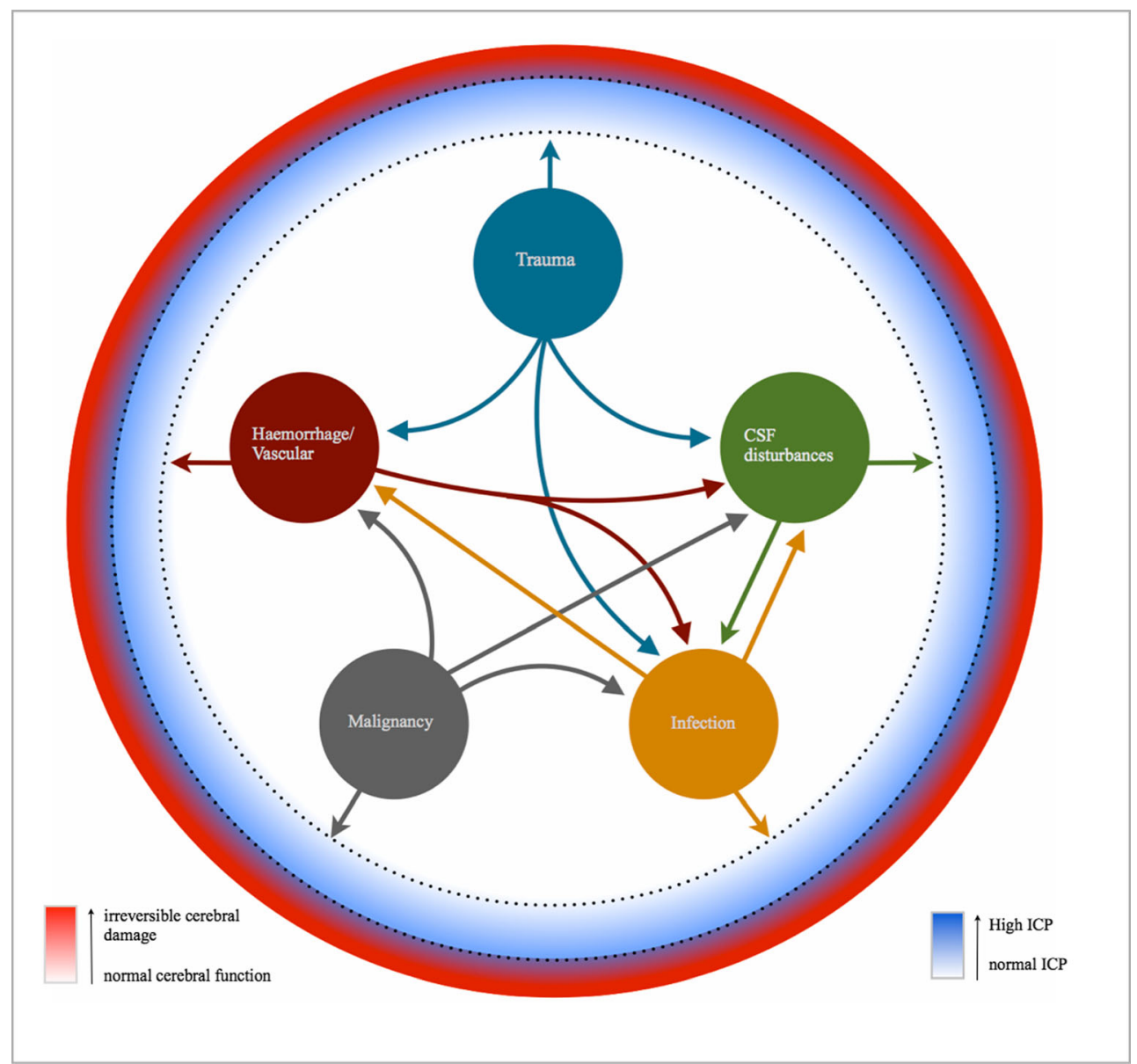

Fig. 5 The relationship between the different intracranial pathological categories and the ICP, cerebral function, RAP index and AMP. Intracranial pathologies can be categorised into five main areas. The large circles represent the initial/primary pathology, with the arrows showing the potential secondary pathologies that can arise from the corresponding initial/primary pathology. For example, trauma can cause a direct increase in the ICP through the expansion of the cerebral parenchyma; however trauma can also result in damage to blood vessels with resulting haemorrhage, or a disturbance within CSF dynamics, e.g., expanding tissue resulting in blockage of the CSF drainage pathways or an increased risk of infection, which could cause a rise in the ICP through increased metabolic turnover. Note that the initial/primary pathology may itself not cause a significant rise in ICP; however the resulting secondary pathologies could be responsible for the rise in ICP, for example a malignant tumour itself may not cause a rise in ICP; however if the surrounding blood vessels rupture then it will be the resulting haemorrhage that will cause the rise in ICP. The nature of the primary pathology will determine what potential secondary pathologies could arise. The dotted circumferential lines represent the interval at which the RAP index and AMP would be most clinically useful. If changes in the AMP and/or RAP index are detected through ICP monitoring before the patient exhibits any signs/symptoms, then this could allow the clinician to implement a change in management sooner and therefore minimise irreversible damage to the brain tissue and subsequently improve patient outcome 
allow the clinician to make an informed decision that will optimise patient outcome, without other factors being involved including patient history, examination findings, basic neuro-imaging, haemodynamic and biochemical markers.

The interval between the onset of raised ICP and just prior to the onset of cerebral damage is where ICP indices could prove most beneficial (Fig. 5).

\section{Conclusion}

Raised ICP is in essence the result of cumulative molecular interactions, which establish three-dimensional multi-variant vectors within the intracranial cavity. This in turn generates pressure gradients that are exacerbated by the rigidity of the encompassing skull.

A sound understanding of this complexity of ICP and how it is affected by intracranial conditions is essential to achieve successful clinical outcome. In addition, as the intracranial cavity is essentially a highly dynamic and organised collection of cells and molecules, the use of mathematical and statistical modelling as a tool to accurately quantify and interpret intracranial dynamics is important.

The important role of minimising disturbances in ICP on a patient's clinical outcome is evident and several studies have improved the ability to use certain ICP parameters to help achieve this. However it remains unclear which of these parameters, specifically, is best in predicting clinical outcome. Both the RAP index and AMP are currently used as markers for certain intracranial physiological parameters; however neither have yet been used as a marker to guide clinical management. Further studies into this potential role of the RAP index and AMP, including current barriers, are warranted.

\begin{abstract}
Acknowledgments We would like to thank Dr Ian Piper, Institute of Neurological Sciences, Queen Elizabeth University Hospital, for providing the ICP recordings used in Figure 1c.

Contributors AH did the literature search, reviewed articles, created all of the figures and wrote the manuscript. RO helped in the writing of the manuscript and critically reviewed the article.
\end{abstract}

\section{Compliance with ethical standards}

Declaration of interest We declare no competing interests.

Funding No funding was received for this research.

Conflict of interest All authors certify that they have no affiliations with or involvement in any organization or entity with any financial interest (such as honoraria; educational grants; participation in speaker's bureaus; membership, employment, consultancies, stock ownership, or other equity interest; any expert testimony or patent licensing arrangements) or non-financial interest (such as personal or professional relationships, affiliations, knowledge or beliefs) in the subject matter or materials discussed in this manuscript.
Ethical approval This study does not contain any studies with human participants or animals performed by any of the authors

Informed consent Informed consent was obtained from all individual participants included in this study.

Animal experiments This study does not contain any studies with human participants or animals performed by any of the authors

Open Access This article is distributed under the terms of the Creative Commons Attribution 4.0 International License (http:// creativecommons.org/licenses/by/4.0/), which permits unrestricted use, distribution, and reproduction in any medium, provided you give appropriate credit to the original author(s) and the source, provide a link to the Creative Commons license, and indicate if changes were made.

\section{References}

1. Czosnyka M, Steiner L, Balestreri M, Schmidt E, Smielewski P, Hutchinson PJ, Pickard JD (2005) Concept of "true ICP" in monitoring and prognostication in head trauma. Acta Neurochir Suppl 95:341-344

2. Kim DJ, Czosnyka Z, Keong N, Radolovich DK, Smielewski P, Sutcliffe MP, Pickard JD, Czosnyka M (2009) Index of compensatory reserve in hydrocephalus. Neurosurgery 64(3):494-501

3. Eide PK, Sorteberg A, Meling TR, Sorteberg W (2014) The effect of baseline pressure errors on an intracranial pressurederived index: results of a prospective observational study. Bio Med Eng 13:99

4. Steiner LA, Andrews PJD. Monitoring the injured brain: ICP and CBF. BJA 97(1) 26-38

5. Budohoski KP, Schmidt B, Smielewski P, Kasprowicz M, Plontke R, Pickard JD, Klingelhöfer J, Czosnyka M (2012) Non-invasively estimated ICP pulse amplitude strongly correlates with outcome after TBI. Acta Neurochir Suppl 114:121-125

6. Czosnyka M, Pickard JD (2004) Monitoring and interpretation of intracranial pressure. J Neurol Neurosurg Psychiatry 75:813-821

7. Krainik A, Hund-Georgiadis M, Zysset S, Yves von Cramon D (2005) Regional impairment of cerebrovascular reactivity and BOLD signal in adults after stroke. Stroke 36(6):1146-1152

8. Zhang L, Yang KH, King AI (2004) A proposed injury threshold for mild traumatic brain injury. J Biomech Eng 126:226-236

9. Hodkinson DJ, O'Daly O, Zunszain PA, Pariante CM, Lazurenko V, Zelaya FO, Howard MA, Williams SCR (2014) Circadian and homeostatic modulation of functional connectivity and regional cerebral blood flow in humans under normal entrained conditions. $\mathrm{J}$ Cereb Blood Flow Metab 34:1493-1499

10. Füchtemeier M, Leithner C, Offenhauser N, Foddis M, Kohl-Bareis M, Dirnagl U, Lindauer U, Royl G (2010) Elevating intracranial pressure reverses the decrease in deoxygenated hemoglobin and abolishes the post-stimulus overshoot upon somatosensory activation in rats. NeuroImage 52(2):445-454

11. Dirven PJ, Haitsma IK, Hoeks SE, Gommers D, Stolker RJ (2015) Ultrasonographic measured optic nerve sheath diameter as an accurate and quick monitor for changes in intracranial pressure. $\mathrm{J}$ Neurosurg 123(3):743-747

12. Kristiansson H, Nissborg E, Bartek J Jr, Andresen M, Reinstrup P, Romner B (2013) Measuring elevated intracranial pressure through noninvasive methods: a review of the literature. J Neurosurg Anesthesiol 25(4):372-385 
13. Avezaat CJJ, Eijndhoven JHM (1984) Cerebrospinal fluid pulse pressure and craniospinal dynamics. A theoretical, clinical and experimental study [Thesis]. The Hague: Jongbloed A

14. Borgesen SE, Gjerris F (1982) The predictive value of conductance to outflow of CSF in normal pressure hydrocephalus. Brain 105: $65-86$

15. Ekstedt J (1977) CSF hydrodynamic studies in man. Method of constant pressure CSF infusion. J Neurol Neurosurg Psychiatry 40:105-119

16. Marmarou A, Shulman K, Rosende RM (1978) A non-linear analysis of CSF system and intracranial pressure dynamics. J Neurosurg 48:332-344

17. Varsos GV, Kasprowicz M, Smielewski P, Czosnyka M (2014) Model-based indices describing cerebrovascular dynamics. Neurocrit Care 20(1):142-157

18. Aries MJ, de Jong SF, van Dijk JM, Regtien J, Depreitere B, Czosnyka M, Smielewski P, Elting JW (2015) Observation of autoregulation indices during ventricular CSF drainage after aneurysmal subarachnoid hemorrhage: a pilot study. Neurocrit Care 23(3): 347-354

19. $\mathrm{Hu}$ X, Xu P, Scalzo F (2009) Morphological clustering and analysis of continuous intracranial pressure. IEEE Trans Biomed Eng 56(3): 696-705

20. Hu X, Xu P, Asgari S, Vespa P, Bergsneider M (2010) Forecasting ICP elevation based on prescient changes of intracranial pressure waveform morphology. IEEE Trans Biomed Eng 57(5):1070-1078 (19)

21. Passi N, Degnan AJ, Levy LM (2013) MR imaging of papilledema and visual pathways: effects of increased intracranial pressure and pathophysiologic mechanisms. AJNR Am J Neuroradiol 34(5): 919-924

22. Ji Z, Zhu L, Yang X, Jiang L (2011) Single wave extraction in continuous intracrania pressure signal with lifting wavelet transformation and discrimination rules. EURASIP J Adv Signal Process 2011:43

23. Ben-Sira L, Goder N, Bassan H, Lifshits S, Assaf Y, Constantini S (2015) Clinical benefits of diffusion tensor imaging in hydrocephalus. J Neurosurg Pediatr 16(2):195-202

24. Carrera E, Kim DJ, Castellani G, Zweifel C, Czosnyka KM, Smielewski P, Pickard JD, Czosnyka M (2010) What shapes pulse amplitude of intracranial pressure? J Neurotrauma 27:317-324

25. Radolovich DK, Aries MJ, Castellani G, Corona A, Lavinio A, Smielewski P, Pickard JD, Czosnyka M (2011) Pulsatile intracranial pressure and cerebral autoregulation after traumatic brain injury. Neurocrit Care 15(3):379-386

26. Eide PK (2005) Assessment of childhood intracranial pressure recordings using a new method of processing intracranial pressure signals. Pediatr Neurosurg 41(3):122-130

27. Holm S, Eide PK (2008) The frequency domain versus time domain methods for processing of intracranial pressure (ICP) signals. Med Eng Phys 30(2):164-170

28. Lee HJ, Jeong EJ, Kim H, Czosnyka M, Kim DJ. (2015) Morphological feature extraction from a continuous intracranial pressure pulse via a peak clustering algorithm. IEEE Trans Biomed Eng

29. Kasprowicz M, Bergsneider M, Czosnyka M, Hu X (2012) Association between ICP pulse waveform morphology and ICP B waves. Acta Neurochir Suppl 114:29-34

30. Eide PK, Sorteberg W (2006) Intracranial pressure and single wave amplitudes, Glasgow Coma Score and Glasgow Outcome Score after subarachnoid haemorrhage. Acta Neurochir (Wein) 148: 1267-1275, 21

31. Eide PK, Sorteberg W (2013) An intracranial pressure-derived index monitored simultaneously from two separate sensors in patients with cerebral bleeds: comparison of findings. Bio Med Eng 12:14
32. Eide PK, Bentsen G, Sorteberg AG, Marthinsen PB, Stubhaug A, Sorteberg W (2011) A randomized and blinded single-center trial comparing the effect of intracranial pressure and intracranial pressure wave amplitude-guided intensive care management on early clinical state and 12-month outcome in patients with aneurysmal subarachnoid hemorrhage. Neurosurgery 69(5):1105-1115

33. Eide PK (2006) Comparison of simultaneous continuous intracranial pressure (ICP) signals from a Codman and a Camino ICP sensor. Med Eng Phys 28(6):542-549

34. Chambers IR, Banister K, Mendelow AD (2001) Intracranial pressure within a developing intracerebral haemorrhage. Br J Neurosurg 15:140-141

35. Fernandes HM, Siddique S, Banister K, Chambers I, Wooldridge T, Gregson B, Mendelow AD (2000) Continuous monitoring of ICP and $\mathrm{CPP}$ following ICH and its relationship to clinical, radiological and surgical parameters. Acta Neurochir Suppl 76:463-466

36. Rosner MJ (1995) Introduction to cerebral perfusion pressure management. Neurosurg Clin N Am 6:761-773

37. Rosner MJ, Daughton S (1990) Cerebral perfusion pressure management in head injury. J Trauma 30:933-940

38. Oertel M, Kelly DF, Lee JH, Glenn TC, Vespa PM, Martin NA (2002) Is CPP therapy beneficial for all patients with high ICP? Acta Neurochir Suppl 81:67-68

39. Lundberg N (1960) Continuous recording and control of ventricular fluid pressure in neurosurgical practice. Acta Psychiatr Scand Suppl 36:1-193

40. Baird TA, Parsons MW, Phanh T, Butcher KS, Desmond PM, Tress BM, Colman PG, Chambers BR, Davis SM (2003) Persistent poststroke hyper-glycemia is independently associated with infarct expansion and worse clinical outcome. Stroke 34:2208-2214

41. Bruno A, Levine SR, Frankel MR, Brott TG, Lin Y, Tilley BC, Lyden PD, Broderick JP, Kwiatkowski TG, Fineberg SE (2002) NINDS rt-PA stroke study group. Admission glucose level and clinical outcomes in the NINDS rt-PA stroke trial. Neurology 59: 669-674

42. Jeremitsky E, Omert LA, Dunham CM, Wilberger J, Rodriguez A (2005) The impact of hyperglycemia on patients with severe head injury. J Trauma 58:47-50

43. Rovlias A, Kotsou S (2000) The influence of hyperglycemia on neurological outcome in patients with severe head injury. Neurosurgery 46:335-342

44. Leigh R, Zaidat OO, Suri MF, Lynch G, Sundararajan S, Sunshine JL, Tarr R, Selman W, Landis DM, Suarez JI (2004) Predictors of hyperacute clinical worsening in ischemic stroke patients receiving thrombolytic therapy. Stroke 35:1903-1907

45. Van den Berghe G, Schoonheydt K, Becx P, Bruyninckx F, Wouters PJ (2005) Insulin therapy protects the central and peripheral nervous system of intensive care patients. Neurology 64:1348-1353

46. Michenfelder JD, Milde JH (1991) The relationship among canine brain temperature, metabolism, and function during hypothermia. Anesthesiology 75:130-136

47. Takagi K (2002) Body temperature in acute stroke. Stroke 33: 2154-2155

48. White H, Cook D, Venkatesh B (2006) The use of hypertonic saline for treating intracranial hypertension after traumatic brain injury. Anesth Analg 102:1836-1846

49. Mangat HS, Chiu YL, Gerber LM, Alimi M, Ghajar J, Härtl R (2015) Hypertonic saline reduces cumulative and daily intracranial pressure burdens after severe traumatic brain injury. J Neurosurg 122(1):202-210

50. Gudeman S, Miller J, Becker D (1979) Failure of high-dose steroid therapy to influence intracranial pressure in patients with severe head injury. J Neurosurg 51:301-306

51. Saul T, Ducker T, Saleman M, Carro E (1981) Steroids in severe head injury: a prospective, randomized clinical trial. J Neurosurg 54:596-600 
52. Saul T, Ducker T, Saleman M, Carro E (1981) Steroids in severe head injury: a prospective, randomized clinical trial. J Neurosurg 54:596-600

53. Feigin VL, Anderson N, Rinkel GJ, Algra A, van Gijn J, Bennett DA (2005) Corticosteroids for aneurysmal subarachnoid haemorrhage and primary intracerebral haemorrhage. Cochrane Database Syst Rev

54. Lozier AP, Sciacca RR, Romagnoli MF, Connolly ES Jr (2002) Ventriculostomy-related infections: a critical review of the literature. Neurosurgery 51:170-181

55. Freeman WD (2015) Management of intracranial pressure. Continuum (Minneap Minn) 21(5 Neurocritical Care):12991323

56. HallowayKL, Barnes T, Choi S, Bullock R,Marshall LF, Eisenberg HM, Jane JA, Ward JD, Young HF, Marmarou A (1996) Ventricolostomy infections: the effect of monitoring duration and catheter exchange in 584 patients. J Neurosurg 85:419-424

57. Schwab S, Georgiadis D, Berrouschot J, Schellinger PD, Graffagnino C, Mayer SA (2001) Feasibility and safety of moderate hypothermia after massive hemispheric infarction. Stroke 32: 2033-2035

58. Dunn L (2002) Raised intracranial pressure. J Neurol Neurosurg Psychiatry 73(Suppl I):i23-i27

59. Juvela S, Heiskanen O, Poranen A, Valtonen S, Kuurne T, Kaste M, Troupp H (1989) The treatment of spontaneous intracerebral hemorrhage: a prospective randomized trial of surgical and conservative treatment. J Neurosurg 70:755-758

60. Mendelow AD, Gregson BA, Fernandes HM, Murray GD, Teasdale GM, Hope DT, Karimi A, Shaw MD, Barer DH (2005) Early surgery versus initial conservative treatment in patients with spontaneous supratentorial intracerebral haematomas in the International Surgical Trial in Intracerebral Haemorrhage (STICH): a randomised trial. Lancet 365:387397

61. Zuccarello M, Brott T, Derex L, Kothari R, Sauerbeck L, Tew J, Van Loveren H, Yeh HS, Tomsick T, Pancioli A, Khoury J, Broderick J (1999) Early surgical treatment for supratentorial intracerebral hemorrhage: a randomized feasibility study. Stroke 30: 1833-1839

62. Czosnyka M, Czosnyka Z, Keong N, Lavinio A, Smielewski P, Momjian S, Schmidt EA, Petrella G, Owler B, Pickard JD (2007) Pulse pressure waveform in hydrocephalus: what it is and what it isn't. Neurosurg Focus 22(4):E1. 11

63. Kase C (1994) Cerebellar hemorrhage. In: Kase CS, Caplan LR (eds) Intracerebral hemorrhage. Mass. Butterworth-Heinemann, Boston, pp 425-443

64. Da Pian R, Bazzan A, Pasqualin A (1984) Surgical versus medical treatment of spontaneous posterior fossa haematomas: a cooperative study on 205 cases. Neurol Res 6:145-151

65. Firsching R, Huber M, Frowein RA (1991) Cerebellar haemorrhage: management and prognosis. Neurosurg Rev 14:191194

66. Kirollos RW, Tyagi AK, Ross SA, van Hille PT, Marks PV (2001) Management of spontaneous cerebellar hematomas: a prospective treatment protocol. Neurosurgery 49:1378-1386

67. Morioka J, Fujii M, Kato S, Fujisawa H, Akimura T, Suzuki M, Kobayashi S (2006) Surgery for spontaneous intracerebral hemorrhage has greater remedial value than conservative therapy. Surg Neurol 65:67-72

68. Sypert G, Arpin-Sypert E (1992) Spontaneous posterior fossa hematomas. In: Kaufman H (ed) Intracerebral hematomas. Raven, New York, pp 187-196

69. Van Loon J, Van Calenbergh F, Goffin J, Plets C (1993) Controversies in the management of spontaneous cerebellar haemorrhage: a consecutive series of 49 cases and review of the literature. Acta Neurochir (Wien) 122:187-193

70. Broderick J, Connolly S, Feldmann E, Hanley D, Kase C, Krieger D, Mayberg M, Morgenstern L, Ogilvy CS, Vespa P, Zuccarello M (2007) Guidelines for the management of spontaneous intracerebral haemorrhage in adults. Stroke 38: 2001-2023

71. Whitfield PC, Patel H, Hutchinson PJ, Czosnyka M, Parry D, Menon D, Pickard JD, Kirkpatrick PJ (2001) Bifrontal decompressive cranietcomy in the management of posttraumatic intracranial hypertension. Br J Neurosurg 15(500-7): 71

72. Hutchinson P, Timofeev I, Kirkpatrick P (2007) Surgery for brain edema. Neurosurg Focus 22(5), E14

73. Wang JW, Li JP, Song YL, Tan K, Wang Y, Li T, Guo P, Li X, Wang Y, Zhao QH (2016) Decompressive craniectomy in neurocritical care. J Clin Neurosci

\section{Comments}

Intracranial pressure is a fundamental signal informing about cerebral homeostasis in the majority of acute and chronic neurological diseases. In neurocritical care it should be interpreted in conjunction with other signals as a part of multimodal brain monitoring. In hydrocephalus or idiopathic intracranial hypertension, ICP is frequently interpreted alone, and additional information may be derived from its pulse waveform, respiratory, composition of slow waves (more or less periodical frequencies slower than $0.05 \mathrm{~Hz}$ ), pathological plateau or 'hyperaemic' elevations. The pulse waveform of ICP has a long list of great publications such as Avezaat and Ejindhoven's PhD thesis (1), Portnoy's arterial pulse to ICP pulse transmission models (2) or the morphological description from the Los Angeles group animated by Xiao Hu (3) and many others. The amplitude of the ICP pulse (AMP) is an effect of pulsation of arterial blood inside compliant cerebral arteries. AMP is roughly proportional to the magnitude of its source (i.e. the amplitude of arterial blood pulsations) and a ratio of compartmental compliances of the cerebral arterial bed and cerebrospinal fluid space. In individual cases the AMP usually increases when the mean ICP increases (4), but only when changes in the mean ICP are not solely procured by changes of central venous pressure (like in a change of body position, but also the Queckenstedt test) or the intracranial pressurevolume curve is very flat (like after craniectomy). In turn, the RAP is a mathematical index derived from changes in the ICP and AMP. As such, it is secondary to the observed changes in the ICP and AMP. Because of the proposed [and verified by so many neuroscientists (5)] linear-exponential shape of the pressure-volume characteristic, it may inform us whether the current working point is on the flat (or linear) part of the curve or exponential (non-linear). In the first case the RAP is close to 0 (signifying the compensatory reserve is good) and in the second close to +1 (the compensatory reserve is exhausted). At very high ICPs, where the pressure-volume curve deflects to the right (5) and decreases its slope (which denotes a critical ICP above which the arterial bed starts to collapse, i.e. the critical closing pressure reaches diastolic blood pressure), the RAP becomes negative.

It is difficult to say which of the two descriptors, AMP or RAP, is more useful. I am sure that both parameters should be analysed in conjunction. High AMP is not always associated with RAP close to +1 . And RAP around 0 is not always associated with low AMP. AMP says more about cerebrovascular phenomena associated with arterial blood transport, and RAP characterises better pressure-volume compensatory reserve. Both may be useful, but RAP cannot be evaluated without proper detection of AMP. And AMP equal to 0 may mean that the patient died, his cerebrospinal 
compliance is abnormally high, or the ICP transducer is broken. References: 1. Avezaat CJJ, van Eijndhoven JHM: Cerebrospinal pulse pressure and craniospinal dynamics. Thesis. A Jongbloed and Zoon Publishers, The Hague, 1984. 2. Portnoy HD, Chopp M, Branch C, Shannon M: Cerebrospinal fluid pulse waveform as an indicator of cerebral autoregulation. J. Neurosurg 56: 666-678, 1982.3. Hu X, Glenn T, Scalzo F, Bergsneider M, Sarkiss C, Martin N, Vespa P. Intracranial pressure pulse morphological features improved detection of decreased cerebral blood flow. Physiol Meas. 2010 May;31(5):679-95.4. Brawanski A, Meixensberger J, Zophel R, Ulrich W: The PA/ICP relationship in head injured patients: is there only one relationship? in JT Hoff, AL Betz (eds): ICP VII. Springer Verlag 1989, p 634-636.5. Lofgren J, von Essen C, Zwetnow NN: The pressure-volume curve of the cerebrospinal fluid space in dogs. Acta Neurol Scand 1973; 49:557-574

Marek Czosnyka

Cambridge UK
1. Avezaat CJJ, van Eijndhoven JHM : Cerebrospinal Pulse Pressure and Craniospinal Dynamics. Thesis. A Jongbloed and Zoon Publishers, The Hague, 1984.

2. Portnoy HD, Chopp M, Branch C, Shannon M: Cerebrospinal fluid pulse waveform as an indicator of cerebral autoregulation. J. Neurosurg 56: 666-678, 1982.

3. Hu X, Glenn T, Scalzo F, Bergsneider M, Sarkiss C, Martin N, Vespa P. Intracranial pressure pulse morphological features improved detection of decreased cerebral blood flow. Physiol Meas. 2010 May;31(5):679-95.

4. Brawanski A, Meixensberger J, Zophel R, Ulrich W: The PA/ICP relationship in head injured patients: is there only one relationship ? in JT Hoff, AL Betz (eds): ICP VII. Springer Verlag 1989, p 634-636.

5. Lofgren J, von Essen C, Zwetnow NN: The pressure-volume curve of the cerebrospinal fluid space in dogs. Acta Neurol Scand1973; 49:557574 